\title{
First Experiments at the ESRF
}

M. Altarelli, one the two Scientific Directors of the European Synchrotron Radiation Facility in Grenoble, reports that while the ESRF's first results to some extent reproduce known results, their quality, scope, and the short time in which they were obtained illustrate the facility's potential.

The ESRF is the first "third generation" synchrotron radiation source to become operational. This generation is characterized by a small size and angular divergence of the electron beam and by the extensive use of insertion devices (wigglers and undulators) as radiation sources, giving an improvement by several orders of magnitude in brilliance of the emitted radiation. The achievement of levels in excess of $10^{17}$ photons $/ \mathrm{s} / \mathrm{mm}^{2}$ / $\mathrm{mrad}^{2} / 0.1 \%$ BW (where BW is the bandwidth) marks the successful completion of the construction and commissioning of the ESRF's accelerator complex.

\section{Micron-Sized Beams Achieved}

The microfocus beam line, designed and built by a group led by $\mathrm{C}$. Riekel, aims to achieve a very small focal spot (less than $\approx 10 \times 10 \mu \mathrm{m}^{2}$ ) for a variety of applications of small and wide-angle scattering and diffraction. The monochromatic source comprises an undulator with a period of $46 \mathrm{~mm}$ and a cryogenically cooled Si channel-cut monochromator.

The ambitious focusing goals of the beam line were achieved and largely surpassed very early in the commissioning of the beam line thanks to the use of Bragg-Fresnel lenses (BFL) as focusing optical elements. Tests of Bragg-Fresnel optics at the ESRF are the results of a long-standing collaboration with the Institute for Microelectronics Technology in Chernogolovka, Russia. The lenses consist of $\mathrm{Si}$ platelets (Fig. 1) on which, using lithographic techniques bor- rowed from microelectronics fabrication technologies, a linear or circular Fresnel grating pattern is engraved. Focussing is obtained upon reflection at the Bragg angle on the platelet (one-dimensional from linear patterns; two-dimensional from circular patterns). Focal lines of $\approx 1 \mu \mathrm{m}$ width, for incident photon energy between 8 and $60 \mathrm{keV}$, were obtained with a BFL of $50 \times 1000 \mu \mathrm{m}^{2}$ with lines down to $0.3 \mu \mathrm{m}$ etched on $\mathrm{Si}$ with a (111) orientation. More recently, a point focus of less than $2 \mu \mathrm{m}$ diameter was achieved by near-back reflection off Si (555) at a wavelength of $1.24 \AA$ using a circular BFL. The resulting focused radiation has been used for small-angle scattering experiments on collagen and other fibres. Sophisticated orientation and alignment techniques allow focusing on a single fibre, with an obvious interest for structural investigations: up to 20 orders of diffraction are clearly resolved in scattering from tendon tissues.

Preliminary results of capillary optics - an alternative to microfocus - are encouraging. In this case, the $\mathrm{x}$-rays enter a $300 \mu \mathrm{m}$ diameter funnel-shaped glass fibre and tumble down by multiple total reflections, to emerge demagnified from the small end of the fibre, giving a beam of about $1 \mu \mathrm{m}$ in diameter.

\section{Broad Scope for Materials Diffraction}

The Materials Science Diffraction beam line, built by group under $\AA$. Kvick, is the first wiggler beam line at ESRF. The source is a 12-periods wiggler, with a $125 \mathrm{~mm}$ period and peak field of $1.24 \mathrm{~T}$. The $5.7 \mathrm{~kW}$ beam

\section{High Brilliance}

Brilliance is the phase-space density of photons, and is defined as the number of photons emitted per unit time by a source of unit area, in a unit solid angle, in a $0.1 \%$ relative bandwidth (BW) around a given frequency. It is customary to express brilliance in units of photons $/ \mathrm{s} / \mathrm{mm}^{2} / \mathrm{mrad}^{2} / 0.1 \%$ BW. The brilliance of ESRF undulators has exceeded $10^{17}$ such units, in a photon energy range from approximately $5 \mathrm{keV}$ to $20 \mathrm{keV}$.

Brilliance is the figure of merit for many x-ray experiments. Qualitatively speaking, high brilliance means that a large number of photons is concentrated in a beam of small dimensions, with a high degree of angular collimation. This allows a high count rate and high momentum resolution in an experiment on a small sample or on a small portion of the sample.

has posed considerable problems of shielding, heat load, and background radiation. It in fact offers the most severe test bench available today for high heat-load $\mathrm{x}$-ray optics so the line has been used to test several monochromators provided by groups from around the world. Both white-beam and monochromatic experiments were performed, the first monochromatic experiments using a watercooled Si crystal in the Laue configuration.

Among the white-beam experiments, energy dispersive diffraction measurements have monitored phase transitions of a $\mathrm{Ca}$ perovskite powder as a function of temperature up to $1900{ }^{\circ} \mathrm{C}$. In the first high-pressure experiment at ESRF, the phases of Ge up to 180 kbar were determined with the help of a diamond anvil cell. Moreover, transitions from the diamond to the $\beta$-tin structures have been clearly identified.
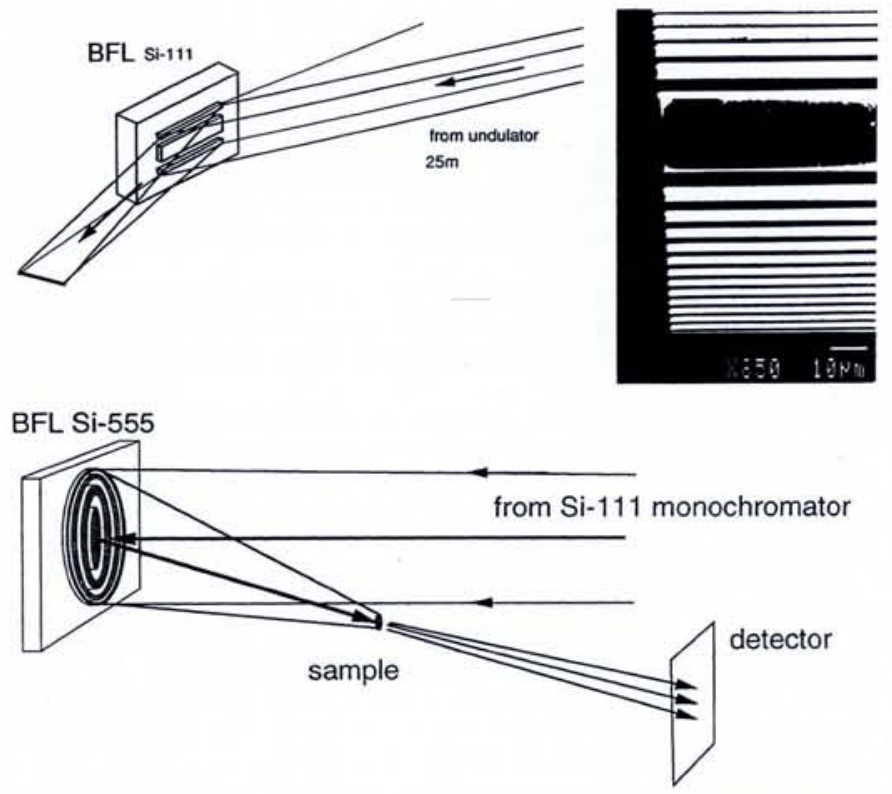

Fig. 1 - Linear (a, upper) and circular (b, lower) Bragg-Fresnel focussing lenses engraved on silicon platelets. The scanning electron micrograph (inset) of the linear pattern shows the sharp groves. The central broad step of the linear lens corresponds to the broad region of non-uniform contrast in the micrograph.

\section{ESRF Beam Line Planning}

The original ESRF planning foresees at least seven beam lines on the floor by mid-1994, and that they should be complete enough to allow calibration experiments. Three lines (1,2 and 9) are already installed; they are well beyond the stage of calibration experiments and are being used for experiments which, although relatively simple and compatible with the requirements of the commissioning phase, are scientifically significant. The hutches of a fourth line are being installed and equipment assembly will start in June, ready for beam acceptance at the end of this summer. Other beam lines will follow with a similar time scale, each shifted from the preceding one by about one month.

A machine diagnostics beam line, mostly intended for machine physics purposes, has been operational since August 1992 and is partially used for other experiments.

In addition to the beam lines built and operated by the ESRF, groups of external laboratories, called Collaborating Research Groups, or CRG's, are building beam lines on bending magnet sources. Agreements with the ESRF allows CRG's to use twothirds of the beam time, while the remaining one-third is contributed to the pool of general user beam time. Two CRG's are presently erecting the hutches of their beam lines, and two more will start to do so this year.

In summary, a dozen beam lines will be visible on the floor by the end of 1993 and most of them will be undergoing commissioning with beams. 


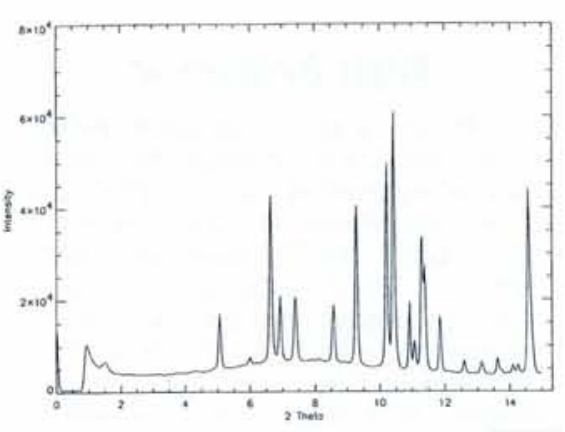

Fig. 2 - A powder diffraction pattern of olivine for a wavelength of $0.45 \AA$ illustrates the high resolution that can be obtained over a wide range of momentum transfer. The angle 2Theta is in degrees.

Monochromatic experiments started with the recording of single-crystal and powder diffraction pattern at energies up to $30 \mathrm{keV}$ $(\approx 0.4 \AA$ wavelength). They allowed the testing of two different two-dimensional positionsensitive detectors - a commercial image plate, and an image intensifier system developed in-house. The powder pattern (Fig. 2) of olivine, a mineral of geophysical interest, recorded at $0.45 \AA$ shows the high resolution that can be obtained over the wide range of momentum transfer allowed by the short wavelength. Furthermore, in the first protein crystallography experiments, complete sets of data on lysozyme and on concanavaline-A were recorded within a few hours, a process that once took days.

The very high critical energy $(\approx 30 \mathrm{keV})$ of the wiggler was used in some other important experiments. White-beam topography experiments revealed the magnetic domain structure of $1 \mathrm{~mm}$ thick Fe samples, thus demonstrating the new capabilities opened up by the penetration of high-energy photons. Compton experiments with $140 \mathrm{keV}$ photons were performed on $\mathrm{LiNbO}_{3}$ in an attempt to detect the charge displacements in this nonlinear dielectric while a large electric field acting on the sample is reversed.

\section{A Versatile Open Undulator}

The Open Undulator beam line was built under the leadership of G. Grübel, following a concept for a multi-station undulator beam line put forward by J. Als-Nielsen. The idea is to interpose thin diamond single crystals in an undulator beam, and to use a Laue reflection from the first crystal to pick out a wavelength for an experiment located sideways, while most of the beam continues on to the next crystal, where another wavelength can be exploited by some other experiment, and so on. In the present configuration of the beam line, only one diamond monochromator is installed, but the versatility of this elegan set-up has already allowed a variety of interesting experiments to be performed.

Protein crystallography has been carried out in both the monochromatic and multiplewavelength anomalous dispersion (MAD) modes. The monochromatic data was taken at $15.75 \mathrm{keV}$ energy, again with a commercial image-plate detector. Systems investigated include a mutant of ornithine carbamoyl transferase, a hydrophobic protein tetramer complex, and seryl-t RNA syntethase on which a total of 102000 reflections were recorded allowing a $2.6 \AA$ resolution (cover illustration)
The MAD method consists in using anomalous diffraction near one of the absorption edges of a specific atom to obtain phase information. An experiment therefore requires scanning from below to above the edge energy. The system investigated by MAD was $\mathrm{N}$-cadherin, which is involved in cell association and binding in the neural system. The part of the molecule studied consists of the first 102 residues, and it binds a $\mathrm{Ca}$ atom which, for the purpose of the experiment, is replaced by $\mathrm{Yb}$.

The $\mathrm{L}_{3}$-edge of $\mathrm{Yb}^{3+}$ near $8.948 \mathrm{keV}$ was chosen. The data, of extremely high quality, are at present under analysis.

A completely different set-up was used for grazing-incidenty diffraction (GID) on monomolecular films on liquid surfaces. LangmuirBlodgett films are formed by long molecules with a hydrophilic head and a hydrophobic tail, which sticks out of the water, often in a straight vertical position. As a function of the density of these monomolecular films on water surfaces, order-disorder and (two-dimensional) crystallization phase transitions can occur. Long-range order is detected by in-plane diffraction of grazing incidence $\mathrm{x}$-ray beams, which emerge in directions different from the one of specular reflection.

Systems investigated were 1-dodecanol on water, which forms a two-dimensional hexagonal lattice below the melting temperature of $39{ }^{\circ} \mathrm{C}$, and $\mathrm{C}_{29} \mathrm{H}_{59} \mathrm{COOH}$, which forms ordered structures where the long molecules are tilted by $27^{\circ}$ with respect to the perpendicular to the water's surface. The first Mössbauer diffraction experiment at ESRF was performed very recently on the open undulator beam line using as the sample a crystal of yttrium-iron garnet isotopically enriched with ${ }^{57} \mathrm{Fe}$. Resonantly diffracted $\mathrm{x}$-rays

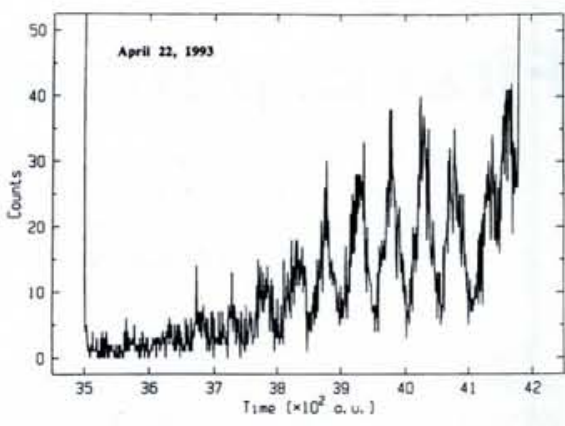

Fig. 3 - Beats in the intensity of nuclear-diffracted $14.4 \mathrm{keV} x$-rays from a yttrium-iron garnet crystal isotopically enriched with ${ }^{57} \mathrm{Fe}$. The resonance of the diffracted $x$-rays corresponds to excitation of extraordinarily narrow nuclear (rather than electronic) levels with widths in the approximately $10^{-8} \mathrm{eV}$ region. The horizontal axis is a time scale running from right to left.

are detected (Fig. 3), where the resonance corresponds to excitation of extraordinarily narrow nuclear (rather than electronic) levels, with widths in the $\approx 10^{-8} \mathrm{eV}$ region.

The intensity of scattered radiation shows characteristic intensity variations in time (beats) due to the proximity in energy of resonances associated to the different hyperfine multiple components. These beats, with period $\approx 10 \mathrm{~ns}$, correspond to $\approx 1 \mu \mathrm{eV}$ energy splitting of the very sharp nuclear resonance of ${ }^{57} \mathrm{Fe}$ near $14.4 \mathrm{keV}$.

\section{ACKNOWLEDGEMENT}

Limitations on space have prevented listing the names of the many people, mostly from the ESRF but also from elsewhere, who deserve full credit for these first results.

\section{KØBENHAVNS UNIVERSITET}

\section{Associate Professorship (lektorat) in Experimental Physics}

A position as associate professor in experimental physics at The Niels Bohr Institute for Astronomy, Physics and Geophysics, Ørsted Laboratory will become vacant on 1 Nov. 1993. Research within the Ørsted Laboratory concentrates on solid state and atomic physics with emphasis on the physics of materials. In particular, research is carried out on III-V heterostructures, the microstructure of materials, chaotic systems, and atomic collision physics. The experimental equipment includes an MBE unit, ion accelerators and implanters, a superconducting $13 \mathrm{~T}$ magnet, a rotating anode $\mathrm{x}$-ray generator, a high-resolution transmission microscope, and Mössbauer spectrometers. Further, a number of lasers and low-temperature equipment are present.

The chosen candidate is expected to contribute to one of the experimental research activities at the Ørsted Laboratory. The associate professor will also participate in university teaching at all levels. The language of instruction is Danish, but English will be accepted for the first two years of the appointment.

Applications should include a curriculum vitae, a complete list of publications, copies of scientific publications, and further documentation, which the applicant wishes to have considered, and a brief outline of proposed research. Information about teaching experience, to be evaluated by the Study Board, should also be enclosed. The material should be submitted in triplicate. After evaluation of the applicants' qualifications by a specially appointed Evaluation Committee, its report will be sent to all applicants. Information about research plans, facilities and staff at the Ørsted Laboratory may be obtained from the Director, Professor Ole Hansen, Niels Bohr Institute for Astronomy, Physics and Geophysics, Blegdamsvej 17, DK-2100 Copenhagen Ø, Denmark; telephone: +4531421616.

The associate professor will be appointed according to existing agreement between the government and the Danish Confederation of Professional Associations. The annual salary will be in the range 276.000 DKr to $323.000 \mathrm{DKr}$ depending on seniority.

Applications marked 221-49/93-5207 L/2-93 which should be written in English, are to be addressed to Rektor of the University of Copenhagen and sent to the Faculty of Natural Sciences, Panum Institute, Blegdamsvej 3, DK-2200 Copenhagen N, Denmark. The closing date for receipt of a fax (fax no.: +45 354310 87) indicating the intention to submit an application is 15 June 1993 (application by 15 July 1993). 